\title{
全氟/多氟烷基化合物在全球海洋水体中的污染演变 趋势研究进展
}

向前，单国强，邬畏，祝凌燕*

南开大学环境科学与工程学院, 环境污染过程与基准教育部重点实验室, 天津城市生态环境修复与污染防治重点实验室, 天津 300350

* 联系人, E-mail: zhuly@nankai.edu.cn

2019-01-09 收稿, 2019-02-23 修回, 2019-02-25 接受, 2019-03-07 网络版发表

国家自然科学基金(21737003, 21577067, 21677081)和高等学校创新引智计划(T2017002)资助

摘要全氟/多氟烷基化合物(PFASs)的环境污染及危害愈来愈受到全球关注。目前, 部分传统PFASs的生产使 用受到限制或管控，一些新型PFASs替代品不断进入市场，使得PFASs的环境污染状况变得更为复杂. PFASs不仅 在人口密集地区的陆地环境中高频检出, 还在各大海洋甚至人迹罕见的两极地区普遍检出. 海洋已经成为PFASs 污染最重要的“汇”之一, 并对全球生态环境以及两极地区产生重要的影响. 关于海洋中PFASs污染的报道也日趋 增多，本文旨在对近年来海洋PFASs污染的研究数据进行剖析，以期阐明PFASs在全球洋流尺度上的污染时空分 布情况，为PFASs的环境风险评估和环境政策的制定提供科学依据.

关键词全氟/多氟烷基化合物，大洋，表层海水，深海海水，洋流

全氟/多氟烷基化合物(per- and poly-fluoroalkyl substances, PFASs) 是一类脂肪烃碳链骨架上部分或 全部氢原子被氟原子取代而形成的人工合成含氟有 机物 ${ }^{[1]}$. 因其优良的化学稳定性、热稳定性、疏水疏 油性和高表面活性等特性, PFASs被广泛应用于电 镀、消防、涂料、造纸、纺织、食品包装等行业 ${ }^{[2]}$. 伴 随其全球规模的生产和使用，在过去十余年间， PFASs造成的环境污染和生态健康风险成为日益关 注的全球性环境问题之一 ${ }^{[3]}$. 其中, 以全氟辛基羧酸 (perfluorooctanoic acid, PFOA)、全氟辛基磺酸 (perfluorooctane sulfonic acid, PFOS)为代表的全氟烷 酸(perfluoroalkyl acids, PFAAs)是环境中检出频率最 高、污染较为突出的PFASs ${ }^{[4,5]}$. 现有的毒理学及流行 病学研究表明, PFAAs具有生物毒性及不良人体健康 效应(如免疫毒性 ${ }^{[6,7]}$ 、致畸性 ${ }^{[6]}$ 、内分泌干扰性 ${ }^{[8,9]}$ 等). 因此, 多国及多个国际组织对于PFAAs的生产
使用做出明确或建议性的限制或禁止 (https://www. federalregister.gov/documents/2013/10/22/2013-24651/ perfluoroalkyl-sulfonates-and-long-chain-perfluoroalkylcarboxylate-chemical-substances-final, http://www.oecd. org/chemicalsafety/risk-management/synthesis-paper-onper-and-polyfluorinated-chemicals.htm). 2009年, PFOS 及其盐类作为限制性化学品被列入斯德哥尔摩公约 附件B(http://www.pops.int/Portals/0/download.aspx ?d=UNEP-POPS-COP.4-SC-4-17.English.pdf)； 2015 年，PFOA及其盐类也被提议纳人斯德哥尔摩公约, 并正在接受进一步的风险评估(http://chm.pops.int/ TheConvention/POPsReviewCommittee/Meetings/POP RC11/POPRC11Followup/tabid/4723/Default.aspx).

纵观目前PFASs的全球环境污染研究, 其污染状 况呈现以下变化趋势：(1) PFASs在环境介质中的污 染以陆源环境介质为主(包括土壤、江河湖水及其底 
泥、生物体等), 逐渐向南北极等偏远区域拓展 ${ }^{[10]}$. 海 洋是陆源污染最重要的“汇”之一 ${ }^{[11]}$, 在PFASs从人类 活动密集区域向极地地区传输的过程中扮演了举足 轻重的角色 ${ }^{[12]}$. 因此, 海洋中PFASs的污染状况受到 越来越多的关注, 其污染范围逐步由近海向远洋发 展 ${ }^{[13]}$. 另外, PFASs的生物累积效应及其涉及的海产

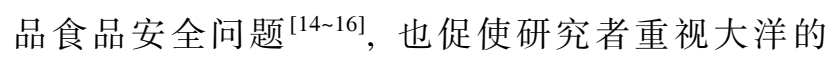
PFAS环境风险. (2) 进人研究视野的PFASs种类逐渐 增多. 因其环境持久性和产地转移等缘故, 较早关注 的PFAAs包括PFCAs $\left(\mathrm{C}_{4 \sim 14}\right)$ 和PFSAs $\left(\mathrm{C}_{4,6,8,10}\right)$, 呈现出 新的时间和空间分布规律 ${ }^{[4,17,18]}$. 由于对以 PFOS, PFOA为代表的中长碳链PFAAs $\left(\mathrm{C}_{n \geqslant 7}\right)$ 的管控, 一些 氟化工业巨头开始使用短碳链PFAAs作为替代, 且 $\mathrm{C}_{4}, \mathrm{C}_{6}$ PFAAs在环境介质中残留水平已经超过PFOS 和PFOA成为主要的全氟污染物, 从而引起研究者的 关注 ${ }^{[17]}$. (3) 在环境中能转化为 PFAAs的前体物质 (PFAA precursors) 包括氟代烷基磺酰氟类 (perfluoroalkyl sulfonyl fluorides, PASFs)、氟调聚物 (fluorotelomers, FTs)、全氟磷酸类 (perfluoroalkyl phosphonic acids, PFPAs)、全氟次磷酸类(perfluoroalkyl phosphinic acids, PFPiAs) 和多氟代磷酸酯 (polyfluoroalkyl phosphate esters, PAPs)等也逐步引起研究 者的关注 ${ }^{[19,20]}$.

综上所述, PFASs 在全球生产和消费的庞大需求 及其环境持久性和长距离传输等特性, 使其在自然环 境中尤其是海洋水体中广泛存在. 目前研究人员针对 PFASs 在全球海洋水体的分布特征已开展了大量研 究, 但这些研究还存在一定的地域局限性, 缺乏对海 洋生态系统中PFASs系统的认识, 亟需回答以下问题: (1) 海洋中PFASs污染在空间和时间上演变有何规律 性?（2) 陆源污染对海洋中PFASs污染有何贡献? (3) 海洋在PFASs全球传输过程中扮演了什么角色? 针对 以上问题, 本文对近年来的研究数据进行了梳理和总 结, 以期阐明PFASs 在全球尺度上的分布特征, 为 PFASs的环境风险评估和环境政策制定提供科学依据.

\section{PFASs 在全球大洋及两极地区海水环境 中的时空分布特征}

\subsection{PFASs在大西洋的时空演变特征}

\subsection{1 污染种类及水平}

2007年之前的监测数据显示, 北大西洋海域和
大西洋赤道附近海域的表层海水中以传统的 PFOA, PFOS为主, 浓度分别为 $10^{2} \sim 10^{3}$ 和 $10^{1} \sim 10^{2} \mathrm{pg} / \mathrm{L}$, 全氟 壬基羧酸(perfluorononanoic acid, PFNA/ $\mathrm{C}_{9} \mathrm{PFCA}$ )、全 氟己基磺酸(perfluorohexane sulfonic acid, $\mathrm{PFHxS} / \mathrm{C}_{6}$ PFSA)和全氟辛基磺胺(perfluorooctane sulfonamide, FOSA) 等其他PFASs 仅少量检出, 浓度介于 $10^{\circ} \sim 10^{1}$ $\mathrm{pg} / \mathrm{L}^{[11,21]}$. 这一时期针对南大西洋表层海水中PFASs 分布特征的研究较少, 仅2004年在安哥拉(Angola)西 部离岸点位 $\left(12^{\circ} 25^{\prime} \mathrm{S} 12^{\circ} 37^{\prime} \mathrm{E}\right)$ 发现了低浓度的PFOA $(<100 \mathrm{pg} / \mathrm{L})^{[11]}$. 2007 2011年, 大西洋绝大部分海域 表层海水中PFOA的浓度有所下降, 但仍达到 $10^{1} \sim 10^{2}$ $\mathrm{pg} / \mathrm{L}$ 水平; PFOS浓度一直稳定在 $10^{1} \sim 10^{2} \mathrm{pg} / \mathrm{L}$ 水平. 同时检出其他种类PFASs, 包括短碳链PFASs(全氟丁 基羧酸(perfluorobutanoic acid, $\mathrm{PFBA} / \mathrm{C}_{4} \mathrm{PFCA}$ )、全氟 戊基羧酸(perfluoropentanoic acid, $\mathrm{PFPeA} / \mathrm{C}_{5} \mathrm{PFCA}$ )、全 氟已基羧酸(perfluorohexanoic acid, PFHxA/ $\mathrm{C}_{6}$ PFCA)、全氟庚基羧酸(perfluoroheptanoic acid, $\mathrm{PFHpA} \mathrm{C}_{7} \mathrm{PFCA}$ )、全氟丁基磺酸(perfluorobutane sulfonic acid, PFBS/C $\mathrm{C}_{4} \mathrm{PFSA}$ )、全氟戊基磺酸 (perfluoropentane sulfonic acid, PFPeS/C 5 PFSA)、 PFHxS、全氟庚基磺酸(perfluoroheptane sulfonic acid, $\left.\mathrm{PFHpS} / \mathrm{C}_{7} \mathrm{PFSA}\right)$ )和长碳链PFCAs(PFNA、全氟癸基羧 酸(perfluorodecanoic acid, PFDA/ $\mathrm{C}_{10} \mathrm{PFCA}$ )、全氟十 一酸(perfluoroundecanoic acid, PFUnDA/ $\mathrm{C}_{11}$ PFCA)、全 氟十二酸(perfluorododecanoic acid, PFDoDA/ $\mathrm{C}_{12}$ PFCA)、全氟十三酸(perfluorotrdecanoic acid, PFTriDA $/ \mathrm{C}_{13} \mathrm{PFCA}$ )、全氟十四酸(perfluorotetradecanoic acid, PFTeDA/C 14 PFCA))等, 浓度均在 $10^{\circ} \sim 10^{2}$ $\mathrm{pg} / \mathrm{L}^{[2225]}$. 值得关注的是, 2009 年之后在西欧近海海 域及河流人海口附近海域检测到新型PFASs, 如中性 PFASs (全氟辛基磺胺醇(perfluorooctane sulfonamidoethanol, FOSE)、全氟丁基磺胺(perfluorobutane sulfonamide, FBSA)、全氟丁基磺胺醇(perfluorobutane sulfonamidoethanol, FBSE)、氟调聚醇(fluorotelomer alcohol, FTOH)、氟调聚丙烯酸酯(fluorotelomer acrylate, FTAC)), 浓度水平在 $10^{-1} \sim 10^{3} \mathrm{pg} / \mathrm{L}$ 范围 内 $^{[26,27]}$, 全氟聚醚类如HFPO-DA浓度水平在 $10^{0} \sim 10^{1}$ $\mathrm{ng} / \mathrm{L}$ 及以下水平 ${ }^{[28,29]}$. 这可能是由于 2000 年以来 $3 \mathrm{M}$, DuPont等氟化工业巨头逐渐停止传统长链PFASs (PFOS, PFOA等)的生产 ${ }^{[30,31]}$, 而又由于PFASs在个 别领域仍然具有不可替代性, PFOS, PFOA及其相关 原料在某些行业依旧具有使用豁免权(https://www. 
federalregister.gov/documents/2013/10/22/2013-24651/ perfluoroalkyl-sulfonates-and-long-chain-perfluoroalkylcarboxylate-chemical-substances-final, http://www.ec. gc. ca/toxiques-toxics/Default.asp? lang=En\&n=96A225B11\#top), 需要更长时间来评估限令实施的效果. 同时 新型PFASs在不断推广, 使得这些区域的PFASs污染 呈现出传统长碳链PFASs和新型PFASs替代品并存的 状况 ${ }^{[32 ~ 34]}$. 目前大西洋远洋海域尚未有上述新型 PFASs 替代品的报道, 但因其稳定的理化性质, 随时 间推移, 这些新型PFASs 可能会随洋流迁移至大西洋 远洋海域 ${ }^{[34]}$.

\subsection{2 海域分布特征}

在大西洋由北往南的表层海水中, PFASs浓度分 布呈现一定的规律性: (1) 南半球海域的PFAAs浓度 总体低于北半球海域. 北大西洋表层海水中的PFOA 和PFOS浓度分别为 $10^{1} \sim 10^{3} \mathrm{pg} / \mathrm{L}$ (美国罗德岛 (Rhode Island)近海岸可达 $10^{0} \sim 10^{1} \mathrm{ng} / \mathrm{L}$ ) 和 $2 \times 10^{1} \sim 2 \times 10^{2} \mathrm{pg} / \mathrm{L}$, 而南大西洋PFOA, PFOS浓度均为 $10^{0} \sim 10^{2} \mathrm{pg} / \mathrm{L}$ (个别 河口点位可达 $\left.1 \times 10^{2} \sim 2 \times 10^{2} \mathrm{pg} / \mathrm{L}\right)^{[22,23,25]}$; (2) PFOA和 $\mathrm{PFOS}$ 二者的浓度比值 $C_{\mathrm{PFOA}} / C_{\mathrm{PFOS}}$ 由北向南逐渐减小. 2007 年以前的研究发现, 北大西洋和大西洋赤道表 层海水中 $C_{\mathrm{PFOA}} / C_{\mathrm{PFOS}}$ 范围分别为4 39和 1 12 ${ }^{[21]} .2009$ 年之后的研究中该比值的海域分布特点更加明显, 在北大西洋 $C_{\mathrm{PFOA}} / C_{\mathrm{PFOS}}>1$, 赤道附近 $C_{\mathrm{PFOA}} / C_{\mathrm{PFOS}}=1$, 南大西洋 $C_{\mathrm{PFOA}} / C_{\mathrm{PFOS}}<1^{[22,23]}$. 上述浓度的南北差异 及 $C_{\mathrm{PFOA}} / C_{\mathrm{PFOS}}$ 比值变化反映了 $\mathrm{PFASs}$ 地域生产使用 的差别: (1) 西欧、北美在大西洋沿海地区工业发达 程度高于南美、西非的大西洋沿海地区, 这些地区排 放的PFASs 经人海河流向大西洋输人, 致使北大西洋 浓度高于南大西洋 ${ }^{[35,36]}$; (2) 自 2002年开始, 北美、欧 洲地区的氟化工业由传统的电氟化法(electrochemical fluorination, ECF) 转向调聚 (telomerization) 合成法. 同时期, 南美洲的国家开始逐渐增加 ECF相关产品 的生产和使用, 造成了南北大西洋表层海水中PFOA 与PFOS的浓度差异 ${ }^{[23,37]}$; (3) 大西洋表层洋流对来自 陆源排放的PFASs的输送和混合, 致使大西洋开放性 海域的PFASs 分布与近海岸海域PFASs 分布紧密相 关, 如南大西洋环流 $\left(C_{\mathrm{PFOA}} / C_{\mathrm{PFOS}}<1\right)$ 与来自北半球的 加那利寒流(Canary Current, $C_{\mathrm{PFOA}} / C_{\mathrm{PFOS}}>1$ ) 在赤道附 近交汇, 促使大西洋赤道附近表层海水中 $C_{\mathrm{PFOA}} /$ $C_{\text {PFOS }}$ 趋近 $1^{[23]}$.

\subsection{3近海和远洋的区域浓度差异对比}

离岸愈近的海域, PFASs浓度呈现上升趋势, 反
之，浓度则呈现下降趋势. 大西洋近北美、南美、西 欧和西非的海域表层海水中, 典型的 PFASs(如 PFOA, PFOS等)浓度比大西洋中部海域高出 1 2 个数 量级. Benskin等人 ${ }^{[23]}$ 发现在美国东部近岸海域(距 Narragansett $330 \mathrm{~km}$ 以内海域), PFOA和PFOS等浓度 较更远海域高 1 个数量级; 在南美洲东北部亚马孙河 (the Amazon) 人海口(河口外675 965 km范围内, https://en.wikipedia.org/wiki/Amazon_River), FOSA浓 度较航线上其他点位高 1 个数量级; 在南美洲东南部 拉普拉塔河(Rio de la Plata)人海口(河口外 65 181 km 以内范围, https://en.wikipedia.org/wiki/Río_de_la_Plata), 典型PFASs(如PFBS, PFHxS, PFOS和PFHxA, PFOA, PFDA, PFUnDA, PFDoDA, PFTriDA和PFTeDA等)的 浓度均比航线上其他远洋点位高 1 个数量级. Ahrens 课题组 ${ }^{[24,25]}$ 和Zhao 等人 ${ }^{[22]}$ 在大西洋东部海域也发现 了相似的规律, PFASs浓度随着与工业污染较为严重 的西欧距离的增加而呈现明显下降趋势，而入海河 流是大西洋海域PFASs 的主要来源 ${ }^{[26,35,36,38 ~ 42]}$. 一些 新型PFASs替代物(如HFPO-DA，全氟乙基环己烷磺 酸(perfluoroethylcyclohexane sulfonate, PFECHS)、全 氟醚基羧酸 (perfluoroalkyl ether carboxylic acids, PFECAs)、全氟醚基磺酸(perfluoroalkyl ether sulfonic acids, PFESAs)等)多出现在大西洋周边地区的人海 河湖水体和生物中或是近岸海水中, 但在远洋海域 尚未发现 ${ }^{[28,29,33,34,43,44]}$.

\subsection{PFASs在太平洋和印度洋海水环境中的时空 分布特征}

\subsection{1 污染种类及浓度水平}

虽然在太平洋近海海域(如东南亚)的调查较为 密集, 但在其远洋海域的监测比大西洋要少. 太平洋 和印度洋远洋表层海水中PFASs的变化趋势与大西 洋相比, 污染物种类相似, 但污染水平稍低一些 ${ }^{[21]}$.

与较早的研究相比 ${ }^{[21]}, 2011$ 年的调查显示 ${ }^{[45]}$ 太 平洋远洋海域表层海水中PFASs种类增多(主要包括 $\mathrm{C}_{4} \sim \mathrm{C}_{10}$ PFCAs, PFHpS, $N$-甲基全氟辛基磺胺 ( $N$-methyl perfluorooctane sulfonamide, $N$-MeFOSA)等), 其中 PFOS浓度(41.1 232 pg/L)增加近1个数量级, PFOA浓 度仍保持在 $2 \times 10^{1} \sim 1 \times 10^{2} \mathrm{pg} / \mathrm{L}$ 水平. 近年来, 西北太 平洋近岸、近海海域发现了除传统PFAAs 和中性 FOSA等以外的一些新型PFASs 替代物, 如 HFPODA、6:2氯代多氟醚磺酸(Cl-6:2 PFESA/F-53B)和 8:2 
氯代多氟醚磺酸(Cl-8:2 PFESA)、短链PFSAs等, 浓度 在 $10^{0} \sim 10^{1} \mathrm{ng} / \mathrm{L}$ 及其以下水平 ${ }^{[28,29,46,47]}$. 这样的变化 与亚洲国家氟化工业生产转型有很大关系. 自 21 世 纪初, 一些北美、欧洲等地区的氟化工业巨头纷纷将 生产线迁往中国、印度等亚洲地区, 导致亚洲地区 PFASs的环境释放量增加 ${ }^{[4,48]}$ (http://www.ihs.com/ products/chemical/planning/ceh/fluoropolymers.aspx?pu= $1 \& \mathrm{rd}=\mathrm{chemihs}$ ). 而早在 20 世纪 70 80年代中国已有 Cl-6:2 PFESA及其同类型产品(如PFOS等)的研发并 投产, 由于它们优良的铬雾抑制效果及其他特性而 广泛应用于工业生产, 但其环境效应当时并未引起 环保人士的关注 ${ }^{[49]}$. 而随着众多国家开始限制PFOS 的生产和使用, 与PFOS结构相似的Cl-6:2 PFESA成 为其替代品, 产量进一步增加, 而环境释放量也随之 增加 ${ }^{[50]}$.

印度洋的数据更少, 仅有的数据显示, 2005 2006年印度洋远洋表层海水中, 传统的 PFOS和 PFOA浓度水平均在 $10^{0} \sim 10^{2} \mathrm{pg} / \mathrm{L}^{[11,51]}$. 2011年印度洋 海域表层海水中 PFASs 种类增加, 包括 FOSA, $N$-MeFOSA. 而传统PFOA, PFOS在该海域表层海水 中的浓度较早期均有升高趋势 (分别为 $2 \times 10^{1} \sim 1 \times 10^{2}$ 和 $\left.10^{1} \sim 10^{3} \mathrm{pg} / \mathrm{L}\right)^{[45]}$.

\subsection{2 海域分布特征}

太平洋的数据显示, PFOA, PFOS在太平洋表层 海水中的分布呈现出西高东低、北高南低的分布形 势. 如2011年PFOA, PFOS浓度水平从西太平洋到东 太平洋, 分别从16 94, 52 232 pg/L下降至13 42, 41.1 216 pg/L, 从北太平洋到南太平洋, 分别从 13 94, 41.1 232 pg/L下降至17 35.1, 95.6 174 pg/L ${ }^{[45]}$. 这主要是由于东亚太平洋沿岸国家和地区(如日本、韩 国、中国)活跃的工业生产排放导致; 而北美地区西 海岸的产业结构偏向农业等, 西部沿海地区鲜有大 型人海河流汇人太平洋, 从而北美西部向太平洋海 域贡献的PFASs排放少; 南半球较少的人类活动(包 括工业活动等)使得南太平洋表层海水中的PFASs污 染也远低于北太平洋.

21 世纪初期, 在太平洋大部分海域呈现出 $C_{\mathrm{PFOA}} / C_{\mathrm{PFOS}}>1^{[11,21]}$, 近年来太平洋远洋大部分海域 该比值已经下降到 1 以下 ${ }^{[45]}$. Chu等人 ${ }^{[52]}$ 发现太平洋 中部中途岛(Midway Island)的鸟类体内发现较高浓 度的长碳链PFASs (如 PFUnDA在肝脏中可以达到 $18.7 \mathrm{ng} / \mathrm{g} \mathrm{ww}$ (湿重)), 并将其归因于这些PFAAs更高
的亲蛋白能力和太平洋垃圾带 (the Great Pacific Garbage Patch, GPGP)带来的塑料垃圾被海鸟误食, 而GPGP受到来自东亚黑潮续流(Kuroshio Extension) 影响较为显著 ${ }^{[33]}$. 这反映了东亚地区氟化工业结构 可能发生改变, 即由早期PFOA大量使用, 到斯德哥 尔摩公约在欧美地区广泛施行后, PFOS类原料及产 品的主产区由欧美地区转移到中国等地区所致.

数据显示, 早期印度洋北部的PFASs污染可能与 近海国家人海河流有关, 如 2005 年斯里兰卡 (Sri Lanka)西部人海河流中PFOS, PFOA可达 $2 \times 10^{1} \sim 1 \times 10^{2}$ $\mathrm{ng} / \mathrm{L}$ 水平 ${ }^{[54]}$. 2005 2006年在印度洋东部和太平洋西 部(菲律宾西部、印度尼西亚哥伦打洛省 (Gorontalo) 北部、南苏拉威西省(South Sulawesi)西南部、澳大利 亚西部和西北部)表层海水中发现PFOS在 $\Sigma$ PFASs中 占比有较大差别(38.6\% 84.6\%), 表明这些海域附近 可能存在不同类型的PFASs 污染源 ${ }^{[51]}$. 而上述东南 亚两国附近点位PFNA占比较大 $(21.4 \%$ 41.5\%), 澳 大利亚西部海域未有PFNA检出, 表明东南亚国家存 在PFNA的潜在污染源 ${ }^{[51]}$. 随着纬度南移, 到与南大 洋交界海域PFASs浓度均显著减少甚至低于检出限 (PFOS: <5 8.6 pg/L, PFOA: 6.4 11 pg/L), 表明印度 洋东南部与南大洋交界海域不存在PFASs 污染源, 且 洋流对PFASs的稀释作用强 ${ }^{[51]}$. 2011年在南印度洋表 层海水中发现由西往东(从南非到澳大利亚), PFOS浓 度逐渐升高(83.8 511 pg/L), 在大洋洲相邻海域达到 峰值, 这可能与澳大利亚的污染排放有关 ${ }^{[45]}$. 综上 所述, 近年来印度洋海域的 PFASs 污染呈现加重 态势.

\subsection{3 近海污染}

太平洋西北部与东亚相接海域存在严重PFASs 污染, 其中以日本和中国近海较为突出. 近年来, 在 PFOA浓度保持基本稳定的同时, PFOS浓度明显减 少, 而短链PFCAs和PFSAs浓度呈上升趋势. 2002年 在日本东京湾、大阪湾表层海水中发现较高浓度 $\left(10^{0} \sim 10^{3} \mathrm{ng} / \mathrm{L}\right)$ 的 PFOS, PFOA, PFHxS，到 2010 年 PFOS及短链PFHxS, PFBS几乎均低于检测限, PFOA 浓度保持稳定, 短链 PFHxA浓度水平可达 $2 \times 10^{2}$ $1 \times 10^{3} \mathrm{ng} / \mathrm{L}^{[55 \sim 57]}$. 在中国的珠江口、渤海湾表层海水 中也发现了类似的规律, 从2003 2016年PFOA浓度 水平一般保持在 $10^{0} \sim 10^{2} \mathrm{ng} / \mathrm{L}$, PFOS 浓度由 $1 \times 10^{1} \sim$ $2 \times 10^{1}$ 下降至 $10^{0} \sim 10^{1} \mathrm{ng} / \mathrm{L}$ 及以下水平, 短链PFAAs浓 度从鲜有检出上升到 $2 \times 10^{1} \sim 1 \times 10^{2} \mathrm{ng} / \mathrm{L}$ 水平, $\mathrm{PFHxS}$ 
浓度一直保持在 $2 \times 10^{1} \mathrm{pg} / \mathrm{L} \sim 2 \times 10^{1} \mathrm{ng} / \mathrm{L}$ 水平 ${ }^{[46,56,58,59]}$. 值得注意的是, 2011 年在中国东海和南海 $35^{\circ} 52.80^{\prime} \sim$ $06^{\circ} 59.40^{\prime} \mathrm{N}$ 范围内, PFOS浓度均低于检测限 $(<0.07$ $\mathrm{ng} / \mathrm{L})^{[60]}$, 而 PFNA在日本人海河流中的检出浓度较 高(最高可达 $2 \times 10^{1} \sim 1 \times 10^{2} \mathrm{ng} / \mathrm{L}$ ), 但是在近岸海域却 鲜有检出 ${ }^{[47]}$. 研究结果表明 ${ }^{[61]}$, PFOS等传统PFASs 被广泛限制使用的背景下, 东亚地区氟化工业生产 结构可能正在发生转变, 即短链PFASs 可能成为替代 品在这些地区广泛使用.

随着与海岸的距离增加, PFASs浓度逐渐降低. 在中国东海到白令海(the Bering Sea)跨 30 个纬度的 海域, 离岸距离 $<135 \mathrm{~km}$ 时, PFOA, PFOS浓度分别为 777 1022，84 105 pg/L, 离岸距离>135 km的海域 PFASs浓度显著减少(其中PFOA, PFOS浓度分别减少 $85 \%, 56 \%$ ), 离岸距离 $>155 \mathrm{~km}$ 时, PFOA, PFOS浓度 水平继续降低并分别稳定在70 80, 10 20 pg/L $\mathrm{L}^{[62]}$. 在中国南海, 离岸距离 $<65 \mathrm{~km}$ 海域, PFOA浓度在 $2 \times 10^{1} \mathrm{pg} / \mathrm{L} \sim 1 \times 10^{1} \mathrm{ng} / \mathrm{L}$ 水平, 其他 PFASs 浓度在 $2 \times 10^{1} \sim 1 \times 10^{3} \mathrm{pg} / \mathrm{L}$ 水平, 离岸距离 $>65 \mathrm{~km}$ 海域, 仅有 低浓度PFOA $(<0.07 \sim 0.13 \mathrm{ng} / \mathrm{L})$ 存在, 再无其他PFASs 检出 ${ }^{[60]}$. 随离岸距离增加, 表层海水中PFASs浓度呈 现下降趋势, 这可能主要是海水稀释效应导致 ${ }^{[45]}$.

\subsection{PFASs 在两极地区海水环境中的时空分布 特征}

2001年Giesy课题组 ${ }^{[10]}$ 首次在南北极动物组织中 发现较高浓度的 PFOS, 其中美国阿拉斯加州 (Alaska)北极熊肝脏中PFOS平均浓度可达 $350 \mathrm{ng} / \mathrm{g}$ ww, 南极洲罗斯海特拉诺瓦湾(Terra Nova Bay)威德 尔海豹(Weddell seal)肝脏中PFOS浓度 $<35 \mathrm{ng} / \mathrm{g} \mathrm{ww}$. 早期对于北冰洋海域的PFASs研究多关注其在雪、大 气等环境介质中的分布特征 ${ }^{[63]}$. 2007年之后的报道 开始关注北冰洋海水中PFAAs的分布特征, 这一时 期的报道发现北冰洋海域表层海水中的PFAAs污染 以传统的 PFAAs 为主, 绝大多数 PFAAs浓度持续保 持在 $2 \times 10^{1} \sim 1 \times 10^{2} \mathrm{pg} / \mathrm{L}$ 及以下水平, 水溶性较高的较 短碳链长度PFCAs普遍浓度高于PFSAs ${ }^{[22,64]}$, 在某些 近岸海域(如格陵兰岛(Greenland)附近海域等), 短链 PFCAs 和FOSA浓度可达 $2 \times 10^{2} \sim 1 \times 10^{3} \mathrm{pg} / \mathrm{L}$ 水平 ${ }^{[65]}$. 在 2007 年之前, 南大洋海域 (the Southern Ocean, 即 $50^{\circ} \mathrm{S}$ 以南的印度洋、大西洋和 $55^{\circ} \sim 62^{\circ} \mathrm{S}$ 之间的太平洋 组成的海域)表层海水中PFASs种类较少(仅有PFOA,
PFOS, PFDoDA检出), 且浓度普遍偏低 $\left(1 \times 10^{1} \sim 2 \times 10^{1}\right.$ $\mathrm{pg} / \mathrm{L}$ 及以下水平 $)^{[51]}$. 直到 2011 年, 南大洋海域的 PFASs 污染种类和浓度较之前并未发生明显变化 ${ }^{[22]}$.

在两极地区, 关于新型PFASs(如Cl-6:2 PFESA, PFECHS, FTSs等)的报道多见于动物体内和北极地 区的大气、雪等环境介质中. 在动物体内, Cl-6:2 PFESA, PFECHS浓度水平可达 $10^{0} \sim 10^{1} \mathrm{ng} / \mathrm{g}$ (脂重)水 平 ${ }^{[66,67]}$; 在北极地区大气中, FTACs, $N$-甲基全氟丁 基磺胺 ( $N$-methyl perfluorobutane sulfonamide, $N$ MeFBSA)、 $N$-甲基全氟丁基磺胺醇 ( $N$-methyl perfluorobutane sulfonamidoethanol, $N$-MeFBSE)的浓度 可达 $1 \times 10^{-1} \sim 1 \times 10^{0} \mathrm{pg} / \mathrm{L}^{[68]}$; 在雪中, FTACs, $N$ MeFBSA, $N$-MeFBSE浓度水平可达 $10^{0} \sim 10^{2} \mathrm{pg} / \mathrm{L}^{[68]}$. 但在两极海水等环境介质中尚未有报道.

在北冰洋海域, 表层海水中传统PFASs浓度均呈 现出西低东高的趋势 ${ }^{[23,69]}$. 以西半球海域为例, PFOA相比PFOS浓度更高, 且 $C_{\mathrm{PFOA}} / C_{\mathrm{PFOS}}$ 比值与经度 存在显著相关性(如加拿大北部北冰洋海域从兰卡斯 特海峡 (Lancaster Sound) 向西到加拿大北极群岛 (Canadian Arctic Archipelago) 以西海域范围内 $)^{[23]}$. 这可能是来自低纬度大西洋海域的海水(其中 $C_{\mathrm{PFOA}} / C_{\mathrm{PFOS}}>1$ ) 随表层洋流向高纬度北冰洋海域传输 的结果, 也可能是由于北美和西欧大气中占主要比 重的FTOH到达该海域上空后发生降解, 然后沉降至 表层海水, 形成PFOA的大气来源 ${ }^{[65,68]}$. 而加拿大北 极群岛西部海域较高的海冰覆盖率, 阻挡了PFASs通 过大气向西传输, 对 $C_{\mathrm{PFOA}} / C_{\mathrm{PFOS}}$ 比值随海域西移而 降低有一定贡献 ${ }^{[23]}$.

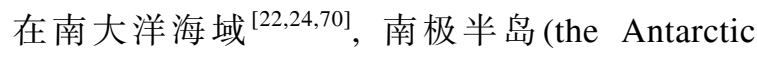
Peninsula)近岸海域表层海水中存在高浓度 $\left(2 \times 10^{2}\right.$ $\left.\mathrm{pg} / \mathrm{L} \sim 1 \times 10^{2} \mathrm{ng} / \mathrm{L}\right)$ 的PFOA, PFHxA, 可能是受当地人 类科考活动的影响. 在大西洋以南的南大洋海域仅 发现低浓度 $\left(2 \times 10^{1} \sim 1 \times 10^{2} \mathrm{pg} / \mathrm{L}\right.$ 及以下) 的 PFOS 和 PFOA, 可能是由于南大洋北部边缘存在南极辐合带 (Antarctic Convergence)和贯穿南大洋大部分海域的 南极绕极流(Antarctic Circumpolar Current)的屏蔽作 用所致，也有可能是通过大气传输而来 ${ }^{[71,72]}$.

\subsection{PFASs在深层海水中的分布特征}

表层海水中PFASs不能全面反映其在海洋环境 中的归趋 ${ }^{[11]}$, 随海水深度的变化, PFASs的分布呈现 一定的特点. 自 2001 年以来, 已在大西洋(探测水深 
范围: 15 5500 m $)^{[11,25]}$ 、太平洋 $(0 \sim 5400 \mathrm{~m})^{[11,55]}$ 、北 冰洋 $(1 \sim 4355 \mathrm{~m})^{[69]}$ 、南大洋 $(0 \sim 3730 \mathrm{~m})^{[11]}$ 等海域开展 了关于PFASs随海洋垂直方向发生迁移的研究.

全球各大洋深层海水中的PFASs 主要由 PFOA, $\operatorname{PFOS}$ 和其他较短碳链的 $\operatorname{PFCAs}\left(\mathrm{C}_{6}, \mathrm{C}_{7}\right)$ 和 $\operatorname{PFSAs}\left(\mathrm{C}_{4}\right.$, $\mathrm{C}_{6}$ ) 组成, 少数海域有 FOSA分布. 在垂直方向上, 随 海水深度增加, PFASs浓度递减, 达到一定深度后(太 平洋西北部约为 1000 1500 $\mathrm{m}^{[55]}$, 大西洋中西部约为 $800 \mathrm{~m}^{[11]}$, 北冰洋海域约为 $150 \mathrm{~m}^{[69]}$ ), PFASs浓度低 于检出限. PFASs 通过沉降流 ${ }^{[25]}$ 和垂直浴流扩散 ${ }^{[73]}$ 等, 或以海水中悬浮颗粒物、生物排泄物为等为载体, 沿纵向向深海传输, 导致深海海水的污染 ${ }^{[74]}$. Yamashita课题组 ${ }^{[11]}$ 在假设汇人北大西洋拉布拉多海中年 均水量恒定的前提下, 估算出该海域表层海水中的 PFOA和PFOS向深海的年均沉积量分别可达 1460 和 $620 \mathrm{~kg}$. 随深度加深, 浓度变低, 是深层海水的稀释 作用造成: 各大洋温跃层 (thermocline) 或盐跃层 (halocline)的下界往往成为阻碍PFASs随下向水流向
更深海域传输的屏障, 而不同大洋温/盐跃层厚度的 差别, 造成PFASs在不同大洋深海传输极限深度的差 异 ${ }^{[11,69]}$. 有趣的是, 在北大西洋拉布拉多海发现 PFASs 浓度随海水深度加深出现先降低后升高的形 势, 研究者解释为是因深海复杂的地形造成的异向 洋流带来的影响 ${ }^{[11]}$.

\section{2 海洋参与的PFASs全球传输机制}

目前研究认为PFASs 在全球大空间尺度上的迁 移有4种途径(图1): (1) 可挥发性、中性前体物质(全 氟烷基磺胺类(perfluoroalkyl sulfonamides, FASAs)、 FTOHs等)通过远距离大气传输(long-range atmospheric transport, LRAT), 到达海洋、极地等偏远地区, 再降解形成PFASs ${ }^{[12,75,76]}$; (2) 可电离的PFASs溶解在 海水中, 并通过洋流的大范围流动而向高纬度偏远 地区(包括北冰洋、南大洋等)迁移 ${ }^{[23,77]}$; (3) 溶解于海 水中的PFASs盐以海洋气溶胶为载体向高纬度偏远 地区传输 ${ }^{[78,79] ; （ 4) ~}$ 在生物体内富集的PFASs 随食物

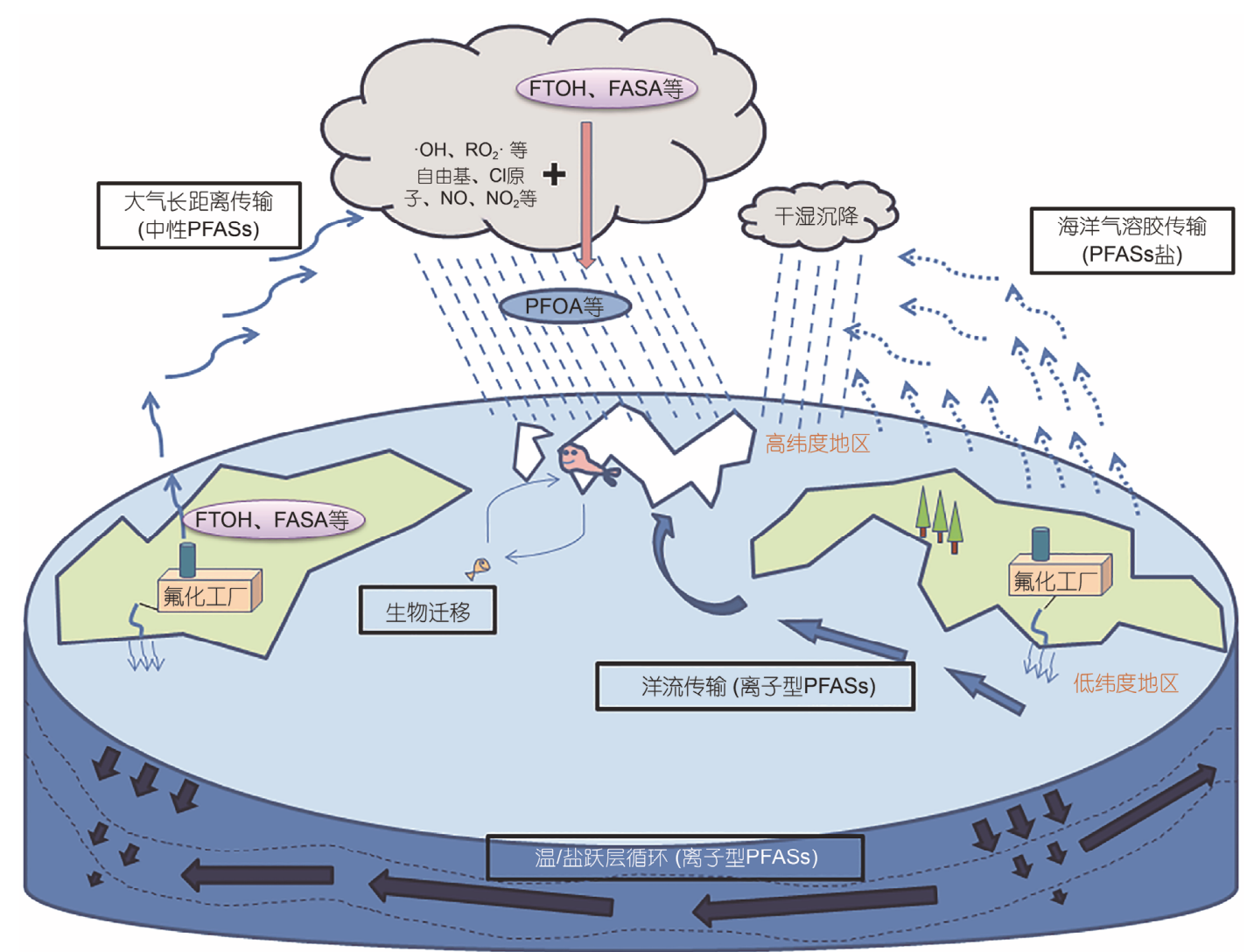

图 1 (网络版彩色)PFASs的几种主要长距离传输机制

Figure 1 (Color online) The main long range transport of PFASs 
链或生物迁徙向高纬度偏远地区传输 ${ }^{[12,72,80]}$.

洋流和海洋气溶胶传输是全氟辛酸盐(perfluorooctanoate, PFO)从低纬度地区向高纬度地区传输的主要 途径. Armitage等人 ${ }^{[77]}$ 通过GloboPOP模型模拟, 显示 洋流的长距离传输方式对北极输送 $\mathrm{PFO}$ 量的贡献大 于FTOH经LRAT后降解的方式. Gomis等人 ${ }^{[81]}$ 通过 OECD Tool模型提出, 亲水性的PFASs替代物(包括 ADONA, HFPO-DA, 全氟三醚羧酸(perfluoro triether carboxylic acid, PFTECA), EEA, 6:2 氟调羧酸 (6:2 fluorotelomer carboxylic acid, 6:2 FTCA)和F-53B等) 通过洋流作用而不是大气传输的方式向偏远地区传 输. $\mathrm{Ti}$ 等人 ${ }^{[82]}$ 同样通过模型 (GloboPOP model)发现 F-53B能通过洋流传输的方式由其主产区/主消费区 如中国向北极地区转移, 且与PFOS有类似的传输特 征, 它们的特征传输距离 (characteristic travel distance)都约为 $1740 \mathrm{~km}$, 由此估算F-53B通过洋流从中 国传输到北极的时间为 1 年多. 因此, 海水及海底沉 积物作为PFASs 全球自然环境中最大的 “汇”, 可能 在更长时间跨度下成为全球PFASs循环的重要环节, 而这又进一步证明了洋流传输作用是高纬度地区 PFASs的主要来源途径. 据估算, 北半球大洋中PFO 通过洋流传输至北极的量约2 12 t/a , 远大于来自北 半球的FTOH通过LRAT-降解-沉降方式向北极传输 PFCAs的量约 $0.1 \sim 1 \mathrm{t} / \mathrm{a}^{[11,12]}$. 此外, Pickard等人 ${ }^{[83]}$ 通 过分析北极冰芯中的PFASs 发现, 大气中的挥发性 PFASs 前体物质通过远距离大气传输-降解-沉降的过 程为北极冰盖贡献了绝大多数的PFAAs, 贡献量远 远大于海洋气溶胶的贡献, 以气溶胶作为载体向高 纬度偏远地区输送PFASs的方式并非北极地区PFASs 的主要来源. 另外, 通过各大洋深海水柱采样分析, 可以看出全球海水的温跃层循环是影响PFASs 向深 海纵向迁移的关键因素 ${ }^{[11,69]}$.

全球气候变暖是影响大气、海洋的PFASs传输机 制的一个重要因素 ${ }^{[84,85]}$. 随着气候变暖, 吸附在极地 海冰中的PFASs 可能随着冰融化效应重新释放到海 水中, 形成PFASs新的来源 ${ }^{[22]}$. 20 世纪已有学者提出 持久性有机污染物 (POPs) 可能通过食物链向南极大 陆传输 ${ }^{[86]}$, 随着研究的深人, Bengtson Nash等人 ${ }^{[72]}$
发现南极迁徙物种如南极半岛的贼鸥(South Polar skua)、白额獲(white-chinned petrel)等在南极寒季时 迁徙到南美洲南部等更温暖处. 这些迁徙鸟类在南 极以外区域暴露和富集PFASs，回迁后通过产蛋、生 物尸体、粪便使其体内的PFASs残留在南极地区. 另 外, 更高营养级的南象海豹(southern elephant seal)受 食范围可由南极近海向北覆盖到几千千米以外的极 锋带(Polar Front Zone), 即南极辐合带的北缘, 它们 也是一种重要的生物载体将PFASs 向南极传输 ${ }^{[72,80]}$. 全球气候变暖可能打破南大洋绕极环流、极锋带等对 南极地区的隔离, 从而促进PFASs以生物迁移方式向 南极传输, 甚至存在早已暴露于PFASs污染的中低纬 度栖息生物向南扩展受食活动范围, 从而向南极地 区输人PFASs 污染 ${ }^{[87]}$. 但目前这种通过生物迁徙方 式向南极地区传输PFASs的量相比洋流传输和LRAT 要少 ${ }^{[80]}$.

\section{3 总结和展望}

在对已有研究成果进行综述的基础上, 对PFASs 在全球海洋水体中的分布研究提出如下思考和展望: (1) 对PFASs在不同空间尺度和时间跨度上的监测调 查亟待加强. PFASs稳定的理化性质和潜在的生态毒 性, 使其对环境的危害风险可能长期存在. 海洋作为 PFASs最大的“汇”，因其覆盖了地球绝大部分的表面 积, 在PFASs的全球迁移归趋中起到重要作用. 而一 些重点海域目前还存在研究空白, 如: 印度洋和北冰 洋东半球海域周边新兴的氟工业主产区(如印度、俄 罗斯等)是PFASs 的潜在污染源. 南大洋作为天然屏 障, 调控PFASs向南极地区传输, 深海具有潜在且巨 大的汇集PFASs的能力, 故函需加强在这些海域中 PFASs的持续性监测, 以期完善对PFASs全球传输机 制的理解. (2) 含氟替代品对海洋生态环境的潜在影 响不容忽视. 如聚醚类、环状PFASs等具有较高生物 富集能力和难降解特性, 虽然目前只在湖泊、河流和 其人海口的海水或者动物体内、沉积物中有发现, 但 从长远来看, 这些物质仍存在向远洋海域迁移的风 险, 故需要持续关注这些含氟替代品在海洋环境的 动态变化.

\section{参考文献}

1 Buck R C, Franklin J, Berger U, et al. Perfluoroalkyl and polyfluoroalkyl substances in the environment: Terminology, classification, and 
origins. Integr Environ Assess Manage, 2011, 7: 513-541

2 Buck R C, Murphy P M, Pabon M. Chemistry, properties, and uses of commercial fluorinated surfactants. In: Knepper T P, Lange F T, eds. Polyfluorinated Chemicals and Transformation Products. Heidelberg: Springer, 2012. 1-24

3 Wang Y W, Cai Y Q, Jiang G B. Research processes of persistent organic pollutants (POPs) newly listed and candidate POPs in Stockholm Convention (in Chinese). Sci Sin Chim, 2010, 40: 99-123 [王亚鞾, 蔡亚岐, 江桂斌. 斯德哥尔摩公约新增持久性有机污染物 的一些研究进展. 中国科学: 化学, 2010, 40: 99-123]

4 Wang Z, Cousins I T, Scheringer M, et al. Global emission inventories for $\mathrm{C}_{4}-\mathrm{C}_{14}$ perfluoroalkyl carboxylic acid (PFCA) homologues from 1951 to 2030, Part I: Production and emissions from quantifiable sources. Environ Int, 2014, 70: 62-75

5 Guo R, Cai Y Q, Jiang G B, et al. Current research of perfluorooctane sulfonate (in Chinese). Prog Chem, 2006, 18: 808-813 [郭睿, 蔡 亚岐, 江桂斌, 等. 全氟辛烷磺酰基化合物(PFOS)的污染现状与研究趋势. 化学进展, 2006, 18: 808-813]

6 Lau C, Anitole K, Hodes C, et al. Perfluoroalkyl acids: A review of monitoring and toxicological findings. Toxicol Sci, 2007, 99: 366-394

7 DeWitt J C, Blossom S J, Schaider L A. Exposure to per-fluoroalkyl and polyfluoroalkyl substances leads to immunotoxicity: Epidemiological and toxicological evidence. J Exposure Sci Environ Epidemiol, 2019, 29: 148-156

8 Shi Z, Zhang H, Ding L, et al. The effect of perfluorododecanonic acid on endocrine status, sex hormones and expression of steroidogenic genes in pubertal female rats. Reprod Toxicol, 2009, 27: 352-359

9 Pedersen K E, Letcher R J, Sonne C, et al. Per- and polyfluoroalkyl substances (PFASs): New endocrine disruptors in polar bears (Ursus maritimus)? Environ Int, 2016, 96: 180-189

10 Giesy J P, Kannan K. Global distribution of perfluorooctane sulfonate in wildlife. Environ Sci Technol, 2001, 35: 1339-1342

11 Yamashita N, Taniyasu S, Petrick G, et al. Perfluorinated acids as novel chemical tracers of global circulation of ocean waters. Chemosphere, 2008, 70: 1247-1255

12 Prevedouros K, Cousins I T, Buck R C, et al. Sources, fate and transport of perfluorocarboxylates. Environ Sci Technol, 2006, 40: 32-44

13 Ju X D. Progress in research on marine environmental pollution of perfluorinated chemicals (in Chinese). Mar Sci, 2010, 34: 93-99 [鞠 晓东. 海洋环境中全氟有机污染物研究的若干进展. 海洋科学, 2010, 34: 93-99]

14 Liu Y, Ruan T, Lin Y, et al. Chlorinated polyfluoroalkyl ether sulfonic acids in marine organisms from Bohai Sea, China: Occurrence, temporal variations, and trophic transfer behavior. Environ Sci Technol, 2017, 51: 4407-4414

15 Zhang H, Lu Y, Luo B, et al. Proteomic analysis of mouse testis reveals perfluorooctanoic acid-induced reproductive dysfunction via direct disturbance of testicular steroidogenic machinery. J Proteome Res, 2014, 13: 3370-3385

16 Liu Y, Richardson E S, Derocher A E, et al. Hundreds of unrecognized halogenated contaminants discovered in polar bear serum. Angew Chem Int Ed, 2018, 57: 16401-16406

17 Wang Z, DeWitt J C, Higgins C P, et al. A never-ending story of per- and polyfluoroalkyl substances (PFASs)? Environ Sci Technol, 2017, 51: 2508-2518

18 Wang Z, Cousins I T, Scheringer M, et al. Global emission inventories for $\mathrm{C}_{4}-\mathrm{C}_{14}$ perfluoroalkyl carboxylic acid (PFCA) homologues from 1951 to 2030, Part II: The remaining pieces of the puzzle. Environ Int, 2014, 69: 166-176

19 Wang Z, Cousins I T, Berger U, et al. Comparative assessment of the environmental hazards of and exposure to perfluoroalkyl phosphonic and phosphinic acids (PFPAs and PFPiAs): Current knowledge, gaps, challenges and research needs. Environ Int, 2016, 89-90: 235-247

20 Wang Z, Cousins I T, Scheringer M, et al. Fluorinated alternatives to long-chain perfluoroalkyl carboxylic acids (PFCAs), perfluoroalkane sulfonic acids (PFSAs) and their potential precursors. Environ Int, 2013, 60: 242-248

21 Yamashita N, Kannan K, Taniyasu S, et al. A global survey of perfluorinated acids in oceans. Mar Pollut Bull, 2005, 51: 658-668

22 Zhao Z, Xie Z, Möller A, et al. Distribution and long-range transport of polyfluoroalkyl substances in the Arctic, Atlantic Ocean and Antarctic coast. Environ Pollut, 2012, 170: 71-77

23 Benskin J P, Muir D C G, Scott B F, et al. Perfluoroalkyl acids in the Atlantic and Canadian Arctic Oceans. Environ Sci Technol, 2012, 46: $5815-5823$

24 Ahrens L, Xie Z, Ebinghaus R. Distribution of perfluoroalkyl compounds in seawater from Northern Europe, Atlantic Ocean, and Southern Ocean. Chemosphere, 2010, 78: 1011-1016

25 Ahrens L, Barber J L, Xie Z, et al. Longitudinal and latitudinal distribution of perfluoroalkyl compounds in the surface water of the Atlantic Ocean. Environ Sci Technol, 2009, 43: 3122-3127

26 Zhao Z, Xie Z, Tang J, et al. Seasonal variations and spatial distributions of perfluoroalkyl substances in the rivers Elbe and lower Weser and the North Sea. Chemosphere, 2015, 129: 118-125

27 Xie Z, Zhao Z, Möller A, et al. Neutral poly- and perfluoroalkyl substances in air and seawater of the North Sea. Environ Sci Pollut Res, 2013, 20: 7988-8000 

11622-11630 isters, 2013 96: 1356-1365 1985, (2): 28-30]

Heydebreck F, Tang J, Xie Z, et al. Correction to alternative and legacy perfluoroalkyl substances: Differences between European and Chinese river/estuary systems. Environ Sci Technol, 2015, 49: 14742-14743

Heydebreck F, Tang J, Xie Z, et al. Alternative and legacy perfluoroalkyl substances: Differences between European and Chinese river/ estuary systems. Environ Sci Technol, 2015, 49: 8386-8395

Posner S, Roos S, Poulsen P B, et al. Per and Polyfluorinated Substances in the Nordic Countries. Copenhagen: Nordic Council of Min-

Ritter S K. Fluorochemicals go short. Chem Eng News, 2010, 88: 12-17

Lin Y F, Ruan T, Jiang G B. Progress on analytical methods and environmental behavior of emerging per- and polyfluoroalkyl substances (in Chinese). Chin Sci Bull, 2017, 62: 2724-2733 [林泳峰, 阮挺, 江桂斌. 新型全氟和多氟烷基化合物的分析、行为与效应研究进 展. 科学通报, 2017, 62: 2724-2733]

Chu S, Letcher R J, McGoldrick D J, et al. A new fluorinated surfactant contaminant in biota: Perfluorobutane sulfonamide in several fish species. Environ Sci Technol, 2016, 50: 669-675

De Silva A O, Spencer C, Scott B F, et al. Detection of a cyclic perfluorinated acid, perfluoroethylcyclohexane sulfonate, in the Great Lakes of North America. Environ Sci Technol, 2011, 45: 8060-8066

Lohmann R, Belkin I M. Organic pollutants and ocean fronts across the Atlantic Ocean: A review. Prog Oceanogr, 2014, 128: 172-184

Stemmler I, Lammel G. Pathways of PFOA to the Arctic: Variabilities and contributions of oceanic currents and atmospheric transport and chemistry sources. Atmos Chem Phys, 2010, 10: 9965-9980

Benskin J P, Ahrens L, Muir D C G, et al. Manufacturing origin of perfluorooctanoate (PFOA) in Atlantic and Canadian Arctic seawater. Environ Sci Technol, 2012, 46: 677-685

Essumang D K, Eshun A, Hogarh J N, et al. Perfluoroalkyl acids (PFAAs) in the Pra and Kakum River basins and associated tap water in Ghana. Sci Total Environ, 2017, 579: 729-735

Ololade I A. Spatial distribution of perfluorooctane sulfonate (PFOS) in major rivers in southwest Nigeria. Toxicol Environ Chem, 2014,

Mudumbi J B N, Ntwampe S K O, Muganza F M, et al. Perfluorooctanoate and perfluorooctane sulfonate in South African river water. Water Sci Technol, 2013, 69: 185-194

Möller A, Ahrens L, Surm R, et al. Distribution and sources of polyfluoroalkyl substances (PFAS) in the River Rhine watershed. Environ Pollut, 2010, 158: 3243-3250

McLachlan M S, Holmström K E, Reth M, et al. Riverine discharge of perfluorinated carboxylates from the European continent. Environ Sci Technol, 2007, 41: 7260-7265

Strynar M, Dagnino S, McMahen R, et al. Identification of novel perfluoroalkyl ether carboxylic acids (PFECAs) and sulfonic acids (PFESAs) in natural waters using accurate mass time-of-flight mass spectrometry (TOFMS). Environ Sci Technol, 2015, 49: tally exposed to perfluorinated compounds. Chemosphere, 2013, 92: 1195-1200

González-Gaya B, Dachs J, Roscales J L, et al. Perfluoroalkylated substances in the global tropical and subtropical surface oceans. Environ Sci Technol, 2014, 48: 13076-13084

Chen H, Han J, Zhang C, et al. Occurrence and seasonal variations of per- and polyfluoroalkyl substances (PFASs) including fluorinated alternatives in rivers, drain outlets and the receiving Bohai Sea of China. Environ Pollut, 2017, 231: 1223-1231

Takemine S, Matsumura C, Yamamoto K, et al. Discharge of perfluorinated compounds from rivers and their influence on the coastal seas of Hyogo prefecture, Japan. Environ Pollut, 2014, 184: 397-404

Li L, Zhai Z, Liu J, et al. Estimating industrial and domestic environmental releases of perfluorooctanoic acid and its salts in China from 2004 to 2012. Chemosphere, 2015, 129: 100-109

Zhu C H. FC-80 chromium for inhibitor (in Chinese). Plating Finishing, 1985, (2): 28-30 [朱楚汉. FC-80 铬雾抑制剂. 电镀与精饰,

Wang S, Huang J, Yang Y, et al. First report of a Chinese PFOS alternative overlooked for 30 years: Its toxicity, persistence, and presence in the environment. Environ Sci Technol, 2013, 47: 10163-10170

Wei S, Chen L Q, Taniyasu S, et al. Distribution of perfluorinated compounds in surface seawaters between Asia and Antarctica. Mar Pollut Bull, 2007, 54: 1813-1818

Chu S, Wang J, Leong G, et al. Perfluoroalkyl sulfonates and carboxylic acids in liver, muscle and adipose tissues of black-footed albatross (Phoebastria nigripes) from Midway Island, North Pacific Ocean. Chemosphere, 2015, 138: 60-66

Lebreton L, Slat B, Ferrari F, et al. Evidence that the Great Pacific Garbage Patch is rapidly accumulating plastic. Sci Rep, 2018, 8: 4666 
54 Guruge K S, Taniyasu S, Yamashita N, et al. Occurrence of perfluorinated acids and fluorotelomers in waters from Sri Lanka. Mar Pollut Bull, 2007, 54: 1667-1672

55 Yamashita N, Kannan K, Taniyasu S, et al. Analysis of perfluorinated acids at parts-per-quadrillion levels in seawater using liquid chromatography-tandem mass spectrometry. Environ Sci Technol, 2004, 38: 5522-5528

56 So M K, Taniyasu S, Yamashita N, et al. Perfluorinated compounds in coastal waters of Hong Kong, South China, and Korea. Environ Sci Technol, 2004, 38: 4056-4063

57 Taniyasu S, Kannan K, Horii Y, et al. A survey of perfluorooctane sulfonate and related perfluorinated organic compounds in water, fish, birds, and humans from Japan. Environ Sci Technol, 2003, 37: 2634-2639

58 Shao M, Ding G, Zhang J, et al. Occurrence and distribution of perfluoroalkyl substances (PFASs) in surface water and bottom water of the Shuangtaizi Estuary, China. Environ Pollut, 2016, 216: 675-681

59 Zhang Y, Lai S, Zhao Z, et al. Spatial distribution of perfluoroalkyl acids in the Pearl River of Southern China. Chemosphere, 2013, 93: $1519-1525$

60 Zheng H, Wang F, Zhao Z, et al. Distribution profiles of per- and poly fluoroalkyl substances (PFASs) and their re-regulation by ocean currents in the East and South China Sea. Mar Pollut Bull, 2017, 125: 481-486

61 Lam J C W, Lyu J, Kwok K Y, et al. Perfluoroalkyl substances (PFASs) in marine mammals from the South China Sea and their temporal changes 2002-2014: Concern for alternatives of PFOS? Environ Sci Technol, 2016, 50: 6728-6736

62 Li L, Zheng H, Wang T, et al. Perfluoroalkyl acids in surface seawater from the North Pacific to the Arctic Ocean: Contamination, distribution and transportation. Environ Pollut, 2018, 238: 168-176

63 Young C J, Furdui V I, Franklin J, et al. Perfluorinated acids in Arctic snow: New evidence for atmospheric formation. Environ Sci Technol, 2007, 41: 3455-3461

64 Rosenberg B, DeLaronde J, MacHutchon A, et al. Spatial and vertical distribution of perfluorinated compounds in Canadian Arctic and sub-Arctic ocean water. Organohalogen Compd, 2008, 70: 386-389

Busch J, Ahrens L, Xie Z, et al. Polyfluoroalkyl compounds in the East Greenland Arctic Ocean. J Environ Monit, 2010, 12: $1242-1246$

mammals. Chemosphere, 2016, 144: 2384-2391

67 Lescord G L, Kidd K A, De Silva A O, et al. Perfluorinated and polyfluorinated compounds in lake food webs from the Canadian high Arctic. Environ Sci Technol, 2015, 49: 2694-2702

68 Xie Z, Wang Z, Mi W, et al. Neutral poly-/perfluoroalkyl substances in air and snow from the Arctic. Sci Rep, 2015, 5: 8912

69 Yeung L W Y, Dassuncao C, Mabury S, et al. Vertical profiles, sources, and transport of PFASs in the Arctic Ocean. Environ Sci Technol, 2017, 51: 6735-6744

70 Cai M, Yang H, Xie Z, et al. Per- and polyfluoroalkyl substances in snow, lake, surface runoff water and coastal seawater in Fildes Peninsula, King George Island, Antarctica. J Hazard Mater, 2012, 209-210: 335-342

71 Corsolini S. Industrial contaminants in Antarctic biota. J Chromatogr A, 2009, 1216: 598-612

72 Bengtson Nash S, Rintoul S R, Kawaguchi S, et al. Perfluorinated compounds in the Antarctic region: Ocean circulation provides prolonged protection from distant sources. Environ Pollut, 2010, 158: 2985-2991

73 Lohmann R, Jurado E, Dijkstra H A, et al. Vertical eddy diffusion as a key mechanism for removing perfluorooctanoic acid (PFOA) from the global surface oceans. Environ Pollut, 2013, 179: 88-94

74 Sanchez-Vidal A, Llorca M, Farré M, et al. Delivery of unprecedented amounts of perfluoroalkyl substances towards the deep-sea. Sci Total Environ, 2015, 526: 41-48

75 Martin J W, Ellis D A, Mabury S A, et al. Atmospheric chemistry of perfluoroalkanesulfonamides: Kinetic and product studies of the OH radical and $\mathrm{Cl}$ atom initiated oxidation of $N$-ethyl perfluorobutanesulfonamide. Environ Sci Technol, 2006, 40: 864-872

76 Ellis D A, Martin J W, De Silva A O, et al. Degradation of fluorotelomer alcohols: A likely atmospheric source of perfluorinated carboxylic acids. Environ Sci Technol, 2004, 38: 3316-3321

77 Armitage J, Cousins I T, Buck R C, et al. Modeling global-scale fate and transport of perfluorooctanoate emitted from direct sources. Environ Sci Technol, 2006, 40: 6969-6975

78 Mader B T. Comment on "Aerosol enrichment of the surfactant PFO and mediation of the water-air transport of gaseous PFOA". Environ Sci Technol, 2009, 43: 1232-1233

79 McMurdo C J, Ellis D A, Webster E, et al. Aerosol enrichment of the surfactant PFO and mediation of the water-air transport of gaseous PFOA. Environ Sci Technol, 2008, 42: 3969-3974

80 Wild S J. Investigating input pathways of persistent organic pollutants to Eastern Antarctica. Doctor Dissertation. Doctor Dissertation. Brisbane: Griffith University, 2016 
81 Gomis M I, Wang Z, Scheringer M, et al. A modeling assessment of the physicochemical properties and environmental fate of emerging and novel per- and polyfluoroalkyl substances. Sci Total Environ, 2015, 505: 981-991

82 Ti B, Li L, Liu J, et al. Global distribution potential and regional environmental risk of F-53B. Sci Total Environ, 2018, 640-641: $1365-1371$

83 Pickard H M, Criscitiello A S, Spencer C, et al. Continuous non-marine inputs of per- and polyfluoroalkyl substances to the High Arctic: A multi-decadal temporal record. Atmos Chem Phys, 2018, 18: 5045-5058

84 Kallenborn R, Halsall C, Dellong M, et al. The influence of climate change on the global distribution and fate processes of anthropogenic persistent organic pollutants. J Environ Monit, 2012, 14: 2854-2869

85 Galbán-Malagón C, Berrojalbiz N, Ojeda M J, et al. The oceanic biological pump modulates the atmospheric transport of persistent organic pollutants to the Arctic. Nat Commun, 2012, 3: 862

86 Sladen W J L, Menzie C M, Reichel W L. DDT residues in Adelie penguins and a crabeater seal from Antarctica. Nature, 1966, 210: 670

87 Fraser C I, Morrison A K, Hogg A M, et al. Antarctica's ecological isolation will be broken by storm-driven dispersal and warming. Nat Clim Change, 2018, 8: 704-708

\section{补充材料}

表 S1＼cjkstart文献中全球各大洋及相关水域水体中 PFASs 浓度分布特征汇总

本文以上补充材料见网络版 csb.scichina.com. 补充材料为作者提供的原始数据, 作者对其学术质量和内容负责. 
Summary for “全氟/多氟烷基化合物在全球海洋水体中的污染演变趋势研究进展”

\title{
The distribution of per- and poly-fluoroalkyl substances in the global marine water
}

\author{
Qian Xiang, Guoqiang Shan, Wei Wu \& Lingyan Zhu*
}

Tianjin Key Laboratory of Environmental Remediation and Pollution Control, Key Laboratory of Pollution Processes and Environmental Criteria of Ministry of Education, College of Environmental Science and Engineering, Nankai University, Tianjin 300350, China

* Corresponding author, E-mail: zhuly@ nankai.edu.cn

Per- and poly-fluoroalkyl substances (PFASs) are a family of artificial organic compounds with at least one hydrogen atom along the carbon backbone substituted with fluorine. They are widely used as surfactants and additives in industries including electroplating, firefighting, coating, textile and so forth because they are chemically and thermally inert and both hydrophobic and oleophobic. PFASs are ubiquitous in various environmental matrix and have caused great concerns on their global transport and adverse impacts on environment. Hence, perfluorooctane sulfonate acid (PFOS) and related substances were listed under Annex B of the Stockholm Convention in 2009. In the recent two decades, an increasingly variety of PFASs are found in the environment (e.g., shorter-chain PFAAs, fluorotelomers, perfluoropolyethers, perfluoroalkyl phosphonic acids, perfluoroalkyl phosphinic acids, polyfluoroalkyl phosphate esters, chlorine or hydro substituted polyfluoroalkyl substances, etc.). They occur not only in inland areas but also in oceans and seas, even in remote areas such as polar areas. This prompts the necessity to figure out the fate of PFASs all over the world. Regarded as one of the crucial sinks of pollutants, oceans and seas play a pivotal role in PFASs transport in the environment. Researches on the marine pollution of PFASs are increasing in the recently years. In this paper, we summarized the recent researches on the spacial and temporal trends of legacy and emerging PFASs in the Atlantic Ocean, the Pacific Ocean, the Indian Ocean, the Arctic Ocean and the Southern Ocean from 2002 to 2016, respectively. We also summarized the researches on the occurrence of PFASs in water at different depths in the middle of the Atlantic Ocean, in the south and northwest of the Pacific Ocean, in the Southern Ocean and in the Arctic Ocean, respectively. The results indicate that a variety of emerging PFASs as substitutes of legacy PFASs are being detected in the oceans, and they are transported from offshore areas to remote areas via currents. The concentrations of PFASs in the seawater decrease gradually with the distance from the coast increasing. The PFASs in the surface seawater of the Arctic Ocean are mainly derived from the North Atlantic Ocean via currents and the degradation of PFASs precursors via atmospheric transport. The occurrence of PFASs in the deep seawater implies that the global thermocline and/or halocline circulation influences the three dimensional transport of PFASs in the oceans and seas. The PFASs transport globally via the following pathways: (1) long-range atmospheric transport (LRAT) plus degradation and precipitation for neutral PFASs precursors; (2) currents and thermocline and/or halocline circulation for ionic PFASs; (3) marine aerosols for perfluorooctanoate; (4) food chain and migratory biota acting as biovectors. In future, more attention should be paid on PFASs in the Indian Ocean and the Southern Ocean, where these is a large data gap on the occurrence of PFASs, which hampers understanding of global transport of PFASs via seas and oceans. Further researches are warranted on the emerging PFAS pollutants in seawater in seas and oceans giving that they are increasingly produced and applied globally.

per- and poly-fluoroalkyl substances, oceans, surface seawater, deep sea water, oceanic currents

doi: 10.1360/N972019-00033 